Reimer, N. K., *Atari, M., *Karimi-Malekabadi, F., Trager, J., Kennedy, B., Graham, J., \& Dehghani, M. (in press). Moral values predict county-level COVID-19 vaccination rates in the United States. American Psychologist. https://doi.org/10.31234/osf.io/z6kxm

\title{
Moral Values Predict \\ County-Level COVID-19 Vaccination Rates in the United States
}

\author{
Nils Karl Reimer ${ }^{1,2}$, Mohammad Atari*1,2, Farzan Karimi-Malekabadi*1,2, Jackson \\ Trager $^{1,2}$, Brendan Kennedy ${ }^{2,3}$, Jesse Graham ${ }^{4}$, Morteza Dehghani ${ }^{1,2,3}$ \\ ${ }^{1}$ Brain and Creativity Institute, University of Southern California \\ ${ }^{2}$ Department of Psychology, University of Southern California \\ ${ }^{3}$ Department of Computer Science, University of Southern California \\ ${ }^{4}$ David Eccles School of Business, University of Utah
}

\begin{abstract}
Author Note
* Atari and Karimi-Malekabadi contributed equally to this work.

Correspondence regarding this article should be addressed to Morteza Dehghani,
\end{abstract} mdehghan@usc.edu, 362 S. McClintock Ave, Los Angeles, CA 90089-161. This research was sponsored by NSF CAREER BCS-1846531, NSF SaTC-2140473 \& DARPA HR001121C0165. We made data and analysis scripts sufficient to reproduce our analyses available online (https://osf.io/vyuhn). This study was not preregistered. We have no known conflict of interest to disclose. 


\begin{abstract}
Despite the widespread availability of COVID-19 vaccines, the United States has a depressed rate of vaccination relative to similar countries. Understanding the psychology of vaccine refusal, particularly the possible sources of variation in vaccine resistance across U.S. sub-populations, can aid in designing effective intervention strategies to increase vaccination across different regions. Here, we demonstrate that county-level moral values (i.e., Care, Fairness, Loyalty, Authority, and Purity) are associated with COVID-19 vaccination rates across 3,106 counties in the contiguous United States. Specifically, in line with our hypothesis, we find that fewer people are vaccinated in counties whose residents prioritize moral concerns about bodily and spiritual purity. Further, we find that stronger endorsements of concerns about fairness and loyalty to the group predict higher vaccination rates. These associations are robust after adjusting for structural barriers to vaccination, the demographic make-up of the counties, and their residents' political voting behavior. Our findings have implications for health communication, intervention strategies based on targeted messaging, and our fundamental understanding of the moral psychology of vaccination hesitancy and behavior.
\end{abstract}

Keywords: Morality; COVID-19; Geo-Spatial Analysis; Vaccine Hesitancy; Moral Foundations Theory 


\section{Public Significance Statement}

Why do people refuse to get vaccinated against COVID-19 even when vaccines are widely available, safe, and efficacious? We show that differences in moral values can help us understand discrepancies in vaccination rates across U.S. counties. Specifically, Purity concerns about bodily and spiritual contamination predicted lower county-level vaccination rates, while Fairness concerns about equality and proportionality and Loyalty concerns about patriotism and ingroup cooperation both predicted higher vaccination rates. 


\section{Moral Values Predict \\ County-Level COVID-19 Vaccination Rates in the United States}

Vaccines save lives. Concerted vaccination programs can pave the way to recovery in global health crises, such as the COVID-19 pandemic. However, despite the fact that safe and efficacious COVID-19 vaccines have been developed, and quick vaccination programs have been implemented globally, these efforts have been hampered by structural inequities (Schoch-Spana et al., 2020) and a lack of access to vaccines in less developed countries (Nkengasong et al., 2020). In addition, skepticism toward vaccination, despite considerable evidence for COVID-19 vaccines being safe (Shimabukuro et al., 2021), limits vaccination programs in some developed countries. In many U.S. regions, especially Republican-voting regions, peoples' concerns regarding the COVID-19 vaccines have delayed the vaccination process (Ivory et al., 2021), resulting in the surge of more deadly variants and preventable fatalities (Mandavilli, 2021). The delay in acceptance, or refusal of vaccines despite the availability of vaccine services, is known as "vaccination hesitancy"

(Dubé et al., 2013) and has often been regarded as one of the most pressing issues in global public health (MacDonald, 2015). In the present work, we show that county-level moral values help explain the stark differences in vaccination rates across the United States, even after controlling for structural barriers and partisan divides.

\section{Structural Barriers to Vaccination}

During the COVID-19 pandemic and after vaccines were developed, many countries had limited access to COVID-19 vaccines. In contrast, the United States has the largest supply of COVID-19 vaccine doses (UNICEF, 2021). Hence, this country provides an ideal context to test how structural barriers and attitudinal variables affect vaccination rates. While vaccine hesitancy is a hurdle to overcome in introducing any new vaccine (De Figueiredo et al., 2020), there may be other reasons why people, who are otherwise willing, do not get vaccinated (Tewarson et al., 2021). Based on existing vaccination programs and early polls on COVID-19 vaccination intent, it can be concluded that 
demand-side challenges persist in the United States (Naterattner, 2021). In addition, the demographic make-up of communities can help explain differences in vaccination rates (Latkin et al., 2021). For example, to examine the relationship between social determinants of health and racial disparities in COVID-19 vaccinations, Agarwal et al. (2021) combined data from state, federal, and other sources and demonstrated racial and other demographic disparities in COVID-19 vaccination rates. To quantify structural and demographic barriers to vaccination rollout, Mishra et al. (2021) created the U.S. COVID-19 Vaccine Coverage Index (CVAC) that identifies which regions and communities may be at risk of poor vaccination coverage. A descriptive examination of this index suggests that states such as Nevada, Mississippi, and Oklahoma are the highest-concern states in the country (Mishra et al., 2021). Failure to understand and address structural barriers to vaccination increases the mortality rate in vulnerable regions and slows down the global progress toward ending the COVID-19 pandemic.

\section{Ideological Barriers to Vaccination}

In addition to structural barriers and demographic characteristics, political ideology is another well-documented determinant of vaccination attitudes and behaviors in the United States. There is compelling evidence that conservatives are less likely to express pro-vaccination beliefs compared with liberals (e.g., Killgore et al., 2021). Moreover, political ideology also has an indirect effect on immunization propensity: ideology predicts trust in government, which in turn predicts attitudes about vaccine choice (Baumgaertner et al., 2018). According to a recent Gallup Poll, Republicans are twice as likely to believe the widely debunked myth that vaccines cause autism (Reinhart, 2020). In addition, exposure to anti-vaccination tweets by former President Trump, who publicly expressed anti-vaccination attitudes, has led to increased concern about vaccines and their safety among his conservative supporters (Hornsey et al., 2020).

A longitudinal survey in the United States covering six months of the COVID-19 
pandemic, from March to August of 2020, showed a decrease in intentions of getting a COVID-19 vaccine when one would become available (Fridman et al., 2021). Furthermore, a decline in general vaccine attitudes and intentions of getting the influenza vaccine was also reported. Follow-up analyses indicated that this substantial decline is driven by participants who identified as Republicans. Indeed, Republicans showed a negative trend in vaccination intentions, while Democrats remained largely stable with regard to their vaccination attitudes. As vaccination efforts continue in the United States during the COVID-19 pandemic, the disparity between Republican-voting and Democratic-voting regions is becoming larger (Ivory et al., 2021), further making COVID-19 vaccination a polarizing matter rather than a politically neutral topic.

\section{Moral Concerns and Vaccination}

Early efforts at studying the effect of various messaging campaigns on vaccine hesitancy has indicated that emotional appeals, rather than information appeals, lower the perceived risk of getting vaccinated (Gursoy et al., 2022). Relatedly, Koinig (2021) found that emotional appeals were preferred by study participants over informational appeals when considering social distancing practices with regard to the COVID-19 pandemic. In addition to emotional appeals, Jung and Albarracn (2021) found that prosocial interventions (i.e., messages that remind individuals about the impact of their being vaccinated on the well-being of others) were, in certain situations, effective in increasing willingness to getting vaccinated. The apparent effectiveness of emotional and prosocial appeals suggest the possibility that attitudes about COVID-19 vaccination are based on deeper intuitions about the vaccine and, furthermore, the people affected by being vaccinated. Specifically, we propose that intuitions about right and wrong - i.e., moral intuitions - are related to vaccine attitudes in the United States and, subsequently, that messaging campaigns might benefit from focusing on moral framing.

Moral judgments and convictions about right and wrong are powerful motivators for 
both prosocial and antisocial behaviors (Graham \& Haidt, 2012; Skitka \& Mullen, 2002). There are currently many psychological theories of the fundamental structure of human morality (e.g. Cushman, 2013; Graham et al., 2013; Gray \& Wegner, 2009; Janoff-Bulman \& Carnes, 2013; Rai \& Fiske, 2011; Schwartz, 1992)ํ․ Moral Foundations Theory (MFT) (Atari et al., 2022; Graham et al., 2013; Haidt \& Joseph, 2004) has sought to expand the range of moral judgments and concerns under scientific scrutiny, combining evolutionary theories with the anthropological work of Richard Shweder (Shweder et al., 1997). MFT posits that a set of innate intuitions lead humans to "gut-level" emotional responses to events in social contexts (Atari, Mostafazadeh Davani, et al., 2020). For example, people have been found to be unable to provide justifications for their decisions regarding moral dilemmas that elicited strong emotional reactions but lacked rational bases (Haidt, 2001).

According to MFT, moral concerns may be reduced to a set of five intuitions. Care, Fairness, Loyalty, Authority, and Purity have been recognized as its core foundations (Graham et al., 2013). Each foundation has developed in response to an evolutionarily and culturally recurrent problem in social living for humans. The foundation of Care is rooted in the instinct to nurture and protect offspring and weak individuals in one's perceived group. Fairness refers to concerns of equality, justice, reciprocity, and proportionality. Loyalty is shaped around concerns of unity, solidarity, togetherness, family, and in-group protection. Authority centers around deference to authority and social order within a hierarchical structure. Finally, Purity relates to physical and spiritual cleanliness, self-discipline, dignity, valuing sacredness, and suppressing carnal desires (Graham et al., 2013).

Related to this paper, conceptually, although vaccines provide a kind of purity-relevant protection against disease, they nevertheless contain elements of the disease

\footnotetext{
1 These theories are complementary in a number of ways (Graham, 2013), but they are generally seen as competitors in the scientific marketplace of ideas. Our goal here is not to discern between the various theories of human morality, but to apply the framework a. that is most relevant to the questions in this project; b. for which data are available to construct the models needed to answer our questions.
} 
and their administration breaks the body membrane, both of which are likely to trigger bodily purity-related concerns. Further, vaccines can be viewed as an unnatural chemical compound, and use of it can be seen as a way to contaminate the body (Hornsey et al., 2018). Other research points to the role of disgust sensitivity in predicting parental vaccine hesitancy, where parents who report higher sensitivity toward disgust show higher hesitancy in getting their kids vaccinated (Reuben et al., 2020). For these reasons, we expected that moral purity concerns would be associated with vaccine hesitancy, rather than acceptance. And this is in fact what was found in the one previous study linking moral foundations to vaccination. In two studies, Amin et al. (2017) found that the relationships between Care and Fairness with vaccine hesitancy (in this case regarding the Human Papillomavirus [HPV] vaccine) were not significant, but that Purity (and Liberty) was significantly associated with vaccine hesitancy (see also replication of this work by Betsch \& Böhm, 2018). ${ }^{2}$ These authors found that, after controlling for age and education, medium-hesitancy parents were twice as likely as low-hesitancy parents to highly endorse Purity. High-hesitancy participants were also twice as likely to strongly emphasize Purity (and Liberty). These findings demonstrate that people's endorsement of Care and Fairness was not reliably predictive of their vaccine hesitancy. Purity (and Liberty), on the other hand, were reliably related to parents' decision to have their children vaccinated against HPV.

Research has shown that there is substantial geographic variation in psychological characteristics and that this variation helps explain regional differences in important real-world outcomes (e.g., Hoover et al., 2021; Jokela et al., 2015). For example, there is strong evidence that U.S. states differ in the aggregated personality traits of their residents (Rentfrow et al., 2008) and these differences are related to differences in social, political, and health-related outcomes (Rentfrow, 2010). Recent research showed that differences in

\footnotetext{
${ }^{2}$ Some MFT theorists have argued that concerns about Liberty may be considered an additional moral foundation (Iyer et al., 2012).
} 
personality traits can also help explain the stark regional differences in COVID-19 transmission in the early stages of the current pandemic (Peters et al., 2020). Similarly, there is evidence that U.S. counties differ in the estimated moral values of their residents and these differences are related to important outcomes such as the prevalence of hate groups (Hoover et al., 2021). However, no studies have tested whether differences in moral values can help explain regional differences in COVID-19 vaccination rates.

\section{The Present Research}

Differences in COVID-19 vaccination rates across U.S. counties are stark — county-level vaccination rates range from $9 \%$ to $96 \%$ - and in need of explanation. Past research has shown that structural barriers (e.g., sociodemographics, healthcare accessibility, resource constrained health systems; Mishra et al., 2021) and political ideologies can delay or prevent vaccination efforts. We argue that, to understand persistent opposition to vaccines despite their health benefits and wide availability, we need to also consider moral values. Past research on the psychological barriers to vaccination has examined vaccine hesitancy and intentions (Amin et al., 2017), but not actual behavior (see Baumeister et al., 2007). In the present research, we investigate whether differences in five moral values (Care, Fairness, Loyalty, Authority, and Purity; Graham et al., 2013) explain differences in COVID-19 vaccination rates across U.S. counties. That is, we relate each county's proportion of residents who have been fully vaccinated to its structural barriers and demographic composition as well as the political leanings and estimated moral values of its residents.

Our overarching prediction is that differences in moral values help explain discrepancies in COVID-19 vaccination rates above and beyond structural, demographic, and political differences. In line with prior research and theorizing, we hypothesize that counties with residents who prioritize Purity concerns have lower vaccination rates. With regard to the other moral foundations, we do not make specific predictions and consider 
our analyses exploratory. Based on a functionalist account of moral foundations, Care and Fairness might be associated with higher vaccination rate, as getting vaccinated helps prevent the spread of the disease to vulnerable groups (such as older or immunocompromised people). Loyalty might be associated with higher vaccination rates if people understand vaccination as an act to protect their ingroup or, alternatively, with lower vaccination rates if people understand vaccination to be in conflict with their ingroup's values or their patriotic duties. Authority might be associated with lower or higher vaccination rates depending on what people understand their leaders' demands to be. ${ }^{3}$

To test our predictions, we combined data on self-reported moral concerns $(106,465$ people) with county-level data on vaccination rates, structural barriers to vaccination, religiosity, and political behavior (3,106 counties). We used geospatial models to estimate vaccination rates in a cross-section of time when vaccines had been widely available in the United States for several months and compare the relative importance of moral values and other factors in predicting vaccination rates.

Our research focuses on county-level differences in vaccination rates rather than on individual-level or state-level differences. We use spatially aggregated rather than individual-level data as doing so allows us to investigate actual vaccination behavior instead of relying on self-reports of vaccination behavior (Baumeister et al., 2007). We use county-level rather than state-level data as county-level data are, in many ways, the most meaningful level of analysis for many psychological, cultural, and political variables in the United States and are the most granular data reported by the Centers for Disease Control and Prevention (CDC). While we control for state-level differences in our analyses, focusing on state-level differences alone would overlook important sub-regional patterns in vaccination rates that do not seem to align with state borders (see Figure 3). Further, we

\footnotetext{
${ }^{3}$ We do not have any county-level data for moral concerns about Liberty, and therefore cannot analyze its relation to vaccination rates.
} 
believe that counties are the most likely and effective entities to enact policies and interventions to shape responses to the pandemic (in California, for example, the San Francisco and Los Angeles counties have set influential COVID-19 policies throughout the pandemic).

\section{Method}

We combined data from several sources to answer our research questions. All observations were at the county level and for the contiguous United States. We made data and scripts for our analyses, as well as results from our analyses, available online (https://osf.io/vyuhn/?view_only=9021343d409f4c64948b61647e17161f). ${ }^{4}$ Table 1 reports descriptive statistics and correlations for measures included in our analyses.

\section{Vaccination Rates}

We operationalized county-level vaccination rates as the cumulative proportion of the total population of a county who had received either both doses of a two-dose vaccine (i.e., the Pfizer-BioNTech or Moderna COVID-19 vaccine) or one dose of a single-dose vaccine (i.e., the Janssen COVID-19 vaccine). We used a dataset that integrates data provided by the Centers for Disease Control and Prevention (CDC) with data provided by State Health Departments to provide accurate estimates of county-level vaccination rates (Tiu et al., 2021). ${ }^{5}$ The CDC data track the numbers of people who are being vaccinated based on information collected from jurisdictional clinics, retail pharmacies, long-term care facilities, dialysis centers, the Federal Emergency Management Agency, Health Resources and Services Administration partner sites, and federal facilities. However, the CDC data

${ }^{4}$ County-level estimates of moral concerns have the potential of being misused for targeting in influence campaigns. To mitigate this risk, we do not share the exact estimates of moral concerns. Instead, we used the 'synthpop' R package (Nowok et al., 2016, Version 1.6-0) to create synthetic data which mimic the original estimates of moral concerns and preserve the relationships with the other variables in the dataset - but sever the link between each county and its residents' moral concerns. All other variables, sufficient to reproduce Models 1 and 2, are provided unaltered.

${ }^{5}$ http://www.vaccinetracking.us 
are missing vaccination rates for some states (e.g., Texas) and counties (e.g., in California) and contains discrepancies with data reported by local health departments. ${ }^{6}$ Tiu et al. (2021) used data from other sources to correct and complete the CDC data. We used the resulting data on the cumulative vaccination rates at a cross-section of time (September 9, 2021) for 3,108 counties in the 48 states and the District of Columbia of the contiguous United States. Vaccination rates ranged from $9 \%$ to $96 \%(M=42 \%, S D=11 \%) .{ }^{7}$

\section{COVID-19 Vaccine Coverage Index}

Mishra et al. (2021) created the U.S. COVID-19 Vaccine Coverage Index (CVAC) based on five themes: (1) historical undervaccination (i.e., below-standard vaccination coverage and high vaccine exemption rate); (2) sociodemographic barriers (i.e., socioeconomic disadvantage, lack of access to information); (3) resource-constrained health system (i.e., limited workforce, infrastructure, and strength); (4) healthcare accessibility barriers (i.e., underinsured, delayed care-seeking, lack of transportation, and limited transit connectivity); and (5) irregular care-seeking behavior (i.e., lack of a personal doctor, failure to seek routine care). The CVAC index was introduced to help identify the community-level barriers affecting vaccine coverage. This index ranks U.S. counties from 0 (lowest concern) to 1 (highest concern) and captures which U.S. communities are at the greatest risk of inadequate vaccination coverage. We used the county-level CVAC index to account for between-county variability of structural predictors in vaccination rates.

\section{Political Ideology}

Since political ideology is related to both COVID-19 vaccination rates (Agarwal et al., 2021) and moral values (Kivikangas et al., 2021), we controlled for the role of political ideology in our statistical models. We collected data from presidential elections in

\footnotetext{
${ }^{6}$ We thank Rex Douglass for bringing these issues to our attention.

${ }^{7}$ We report analyses using the original, unaltered, CDC data in the Supplemental Materials, with qualitatively similar findings.
} 
the U.S. (MIT Election Data and Science Lab, 2018) and calculated a county-level conservatism index by subtracting the votes for the Democratic party from the votes for the Republican party and dividing that quantity by the total votes (including for the Green party) in that county across the 2000-2020 presidential elections.

\section{Endorsement of Moral Values}

We used the estimates of county-level distribution of moral values provided by Hoover et al. (2021). These authors relied on data from the crowd-sourcing website www.YourMorals.org, which is an online platform for collecting a wide range of psychological data, including moral values. These county-level estimates are based on data from 2012 to 2018 ( $N=106,465)$ using the Moral Foundations Questionnaire (MFQ; Graham et al., 2011). The MFQ measures to what extent respondents endorse concerns about caring for others and preventing harm to the weak and vulnerable (Care); about fairness and equality (Fairness); about being loyal to one's family, country, and other ingroups (Loyalty); about respecting hierarchies and protecting traditions (Authority); and about avoiding indecent, impure, or unnatural acts (Purity). ${ }^{8}$

Although this is a large sample, it cannot be used to directly estimate county-level moral values as it does not use probability sampling at the county level (Hoover \& Dehghani, 2019), and many counties have very few respondents. To address these issues, Hoover et al. (2021) used Multilevel Regression and Synthetic Poststratification (MrsP; Leemann \& Wasserfallen, 2017), a model-based approach for sub-national estimation that extends Multilevel Regression and Poststratification (MrP; Park et al., 2004). The multilevel model also included a hierarchical auto-regressive prior (Riebler et al., 2016) that, under the presence of spatial autocorrelation, induces local spatial smoothing between proximate counties (Hanretty et al., 2016; Hoover \& Dehghani, 2019). To validate the county-level estimates of moral concerns, Hoover et al. (2021) analyzed the relation between

\footnotetext{
${ }^{8}$ Further details and example items are provided in the supplemental materials.
} 


\section{Table 1}

Correlations, as well as means and standard deviations, for all county-level variables included in the main analyses

\begin{tabular}{|c|c|c|c|c|c|c|c|c|c|c|c|c|}
\hline \multirow[b]{2}{*}{$\#$} & \multirow[b]{2}{*}{ Measure } & \multirow[b]{2}{*}{$M$} & \multirow[b]{2}{*}{$S D$} & \multicolumn{9}{|c|}{$r$} \\
\hline & & & & 1 & 2 & 3 & 4 & 5 & 6 & 7 & 8 & 9 \\
\hline 1 & $\%$ vaccinated & 0.42 & 0.11 & & -.01 & -.42 & -.62 & .45 & .53 & -.61 & -.63 & -.71 \\
\hline 2 & $\%$ over 65 & 0.20 & 0.05 & & & -.11 & .17 & -.19 & -.26 & .20 & .14 & .18 \\
\hline 3 & CVAC Index & 0.50 & 0.29 & & & & .13 & .01 & .07 & .36 & .51 & .45 \\
\hline 4 & Conservatism & 0.23 & 0.27 & & & & & -.55 & -.82 & .75 & .57 & .75 \\
\hline 5 & Care & 3.45 & 0.05 & & & & & & .80 & -.33 & -.31 & -.41 \\
\hline 6 & Fairness & 3.35 & 0.08 & & & & & & & -.58 & -.38 & -.58 \\
\hline 7 & Loyalty & 2.91 & 0.09 & & & & & & & & .89 & .91 \\
\hline 8 & Authority & 3.15 & 0.12 & & & & & & & & & .94 \\
\hline 9 & Purity & 2.92 & 0.21 & & & & & & & & & \\
\hline
\end{tabular}

MFQ estimates of county-level proportion of conservatives against ground-truth data (i.e. 2016 share of Republican votes by estimates of county-level proportion). They report a substantial correlation $(r=0.63,95 \% \mathrm{CI}=[0.60,0.65])$ between the estimated measure and the observed, ground truth measure of ideological distribution at the county-level, further validating the county-level estimates produced by MrsP. Other research has validated the estimates produced by Multilevel Regression and Poststratification techniques across different data domains (e.g. Hoover \& Dehghani, 2019; Wang et al., 2015).

The resulting estimates of county-level moral values can be viewed at mapyourmorals.usc.edu as well as in Figure 1, which shows the geographic distributions of the estimated county-level moral values and of county-level conservatism.

\section{Additional Control Variables}

In addition to the ones used in the main analyses, we considered a broader range of measures as control variables in the robustness checks. First, we included county-level demographics characteristics from the 2019 American Community Survey and, where available, the 2020 Decennial Census (Walker \& Herman, 2021): the (log-transformed) median income; the proportion of residents with a bachelors degree or higher; the 


\section{Figure 1}

Estimates of each county's residents' endorsement of moral concerns and political ideology
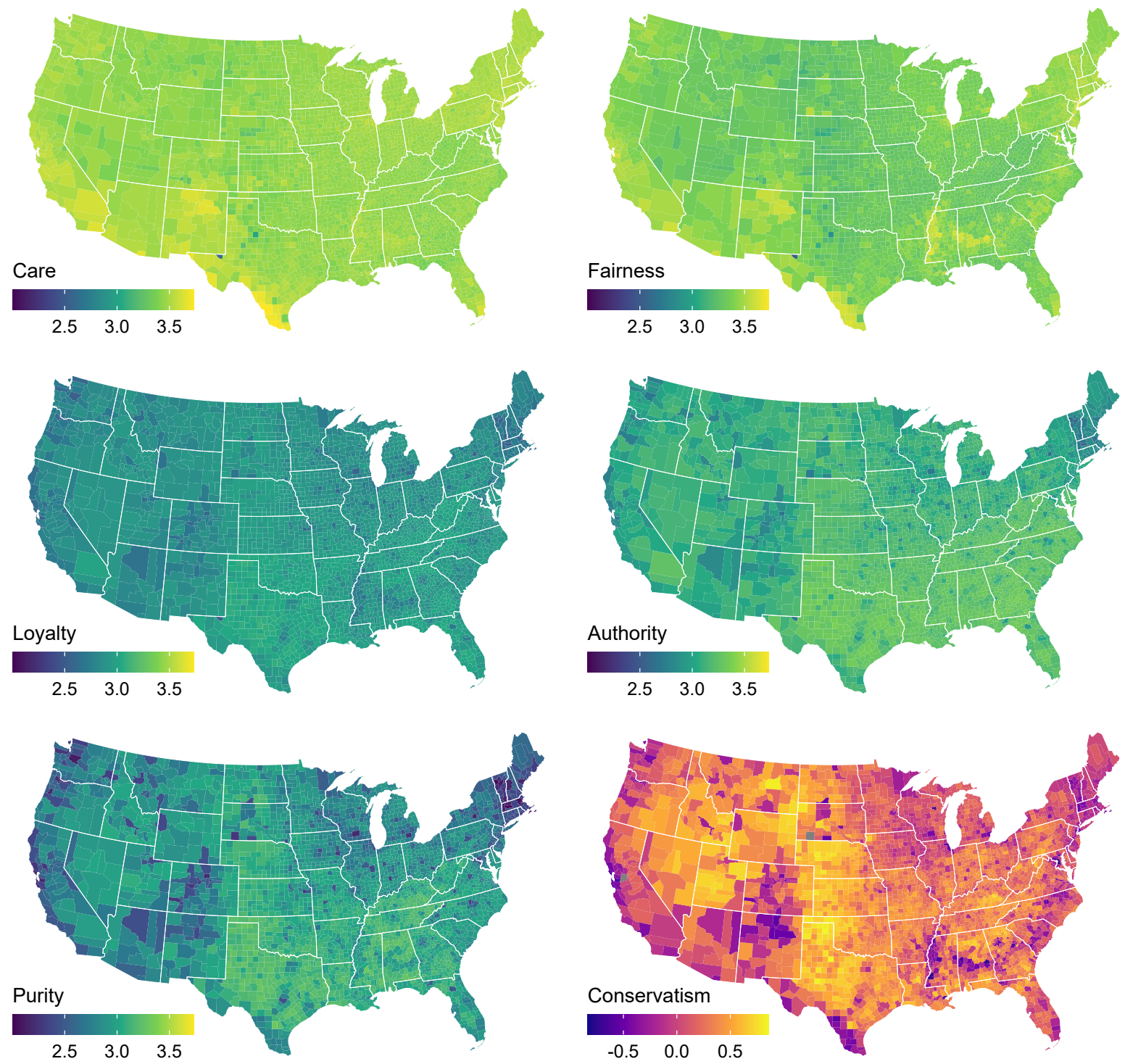

Note. For an interactive visualization of county-level moral values, see mapyourmorals.usc.edu. 
(log-transformed) population density; and the proportions of White non-Latino, Black non-Latino, and Latino residents. Second, we re-calculated the conservatism index using only data from the 2020 presidential election to distinguish the contribution of long-running political tendencies from the partisan politics of the presidential election held during the pandemic. Third, we included county-level rates of religious adherence based on data from the 2010 U.S. Religion Census (Grammich et al., 2010). Finally, we considered partial vaccination rates from Tiu et al.'s 2021 data set - that is, the county-level proportion of residents who received at least one dose of a vaccine - as an alternative outcome variable. As these data were not consistently reported by Nebraska and South Dakota, analyses focusing on partial vaccination rates excluded those states.

\section{Analytic Strategy}

Vaccination rates are proportions and, as such, bounded between 0 and 1 . We used beta regression with a logit link function (Ferrari \& Cribari-Neto, 2004) to estimate county-level vaccination rates as a function of relevant predictor variables. In beta regression, the intercept represents the average log odds of vaccination when all predictor variables are zero. Regression coefficients are log odds ratios and represent the change in the log odds of vaccination for each one unit increase in the predictor variable. We standardized all predictor variables. We transformed regression coefficients to odds ratios so that they represent the estimated change in the average odds of vaccination for each $1 S D$ increase in the predictor variable. The odds of vaccination, in turn, can be converted to vaccination rates (e.g., odds of 1 correspond to a $50 \%$ vaccination rate). Our models included a spatial autocorrelation component to account for the tendency of neighboring counties to resemble each other and a non-spatial component to capture residual variation across counties (Morris et al., 2019; Riebler et al., 2016). Our models used an adjacency matrix that encoded, for each pair of counties, whether they share a border (1) or not (0). Our models also included a varying (random) intercept to account for differences in 
policies, resources, and other factors between states (and the District of Columbia) that were not captured by the control variables.

To estimate these models, we used the 'brms' R package (Bürkner, 2017, 2018) as an interface to fit Bayesian generalized linear multilevel models in Stan (Stan Development Team, 2021). Bayesian inference involves choosing a likelihood function and prior distributions. The likelihood function links the observed data to one or more model parameters (e.g., regression coefficients) by expressing how likely the observed data would have been for different values of said model parameters. Our models used a beta likelihood with a logit link function. Prior distributions state how plausible different values of said model parameters are before considering the observed data. Our models used weakly informative prior distributions, $\beta \sim \operatorname{Student-t}(3,0,2.5)$ on the log odds scale, for the fixed intercept, the regression coefficients, and the standard deviation of the varying intercept. Bayesian inference applies Bayes' theorem to update prior distributions in light of the observed data to produce posterior distributions. Posterior distributions state how plausible different values of the model parameters are given the observed data. We report point estimates, based on the median of posterior samples, and 95\% uncertainty intervals, based on the quantiles of posterior samples, for relevant model parameters.

To compare the relative importance of structural, political, and moral variables for predicting vaccination rates, we compared a series of models. Model 1 included only the proportion of residents over 65 and the CVAC index as predictor variables. We controlled for the proportion of residents over 65 because this age group both had earlier access to vaccination in most states and is at a higher risk of serious illness or death after infection. Model 2 included political ideology as an additional predictor variable. Model 3 included all predictor variables from previous models as well as county-level estimates of moral values.

We used 10-fold cross-validation to estimate how well each model predicted observations outside the sample used to estimate it. As a measure of out-of-sample prediction accuracy, we calculated each model's expected log predictive density $(E L P D)$, 
that is, the logarithm of the joint posterior predictive probability of all observations. To compare models, we calculated the difference in out-of-sample prediction accuracy for each pair of models $\left(\Delta_{E L P D}\right)$, with positive values indicating that a model made more accurate predictions than a comparison model. ${ }^{9}$ We divided this difference by its standard error $\left(z=\Delta_{E L P D} / S E\right)$ to account for the uncertainty of cross-validation as an estimate of out-of-sample prediction accuracy (Vehtari et al., 2017). We selected a more complex over a simpler model when the difference in prediction accuracy was much larger than its standard error. ${ }^{10}$ In addition, we report a Bayesian analogue of $R^{2}$ with its $95 \%$ uncertainty interval (Gelman et al., 2019) as a measure of within-sample model fit.

\section{Results}

We report results from our main analyses investigating the relative importance of demographic, structural, political, and moral differences for predicting country-level vaccination rates as well as results from additional analyses investigating how robust our findings were to different analytic choices.

\section{Main Analyses}

Figure 2 shows the results of our main analyses. Model 1, which included only structural and demographic predictor variables, explained $R^{2}=19 \%,[16 \%, 22 \%]$ of variance in vaccination rates. Both the CVAC index $(O R=0.82,[0.80,0.84])$ and the proportion of residents over $65(O R=0.97,[0.96,0.98])$ were associated with lower odds of vaccination. ${ }^{11}$ Model 2, which included partisanship as an additional predictor variable,

\footnotetext{
${ }^{9}$ Spatial autoregressive models are non-generative models, that is, they cannot make predictions for areas not included in the model. To still compare the relative prediction accuracies of our models, we used only the non-spatial, fixed effects to estimate each model's out-of-sample prediction accuracy. Likewise, we only used the non-spatial, fixed effects to estimate $R^{2}$ values.

${ }^{10}$ For a conventional interpretation, consider the critical values to be $\left|\Delta_{E L P D} / S E\right|>1.96$ for $p<.05 ; 2.58$ for $p<.01$; and 3.29 for $p<.001$ in a two-sided null-hypothesis significance test of the difference in out-of-sample prediction accuracy.

${ }^{11}$ Readers might be surprised to read that the proportion of residents over 65 is associated with lower vaccination rates in a county since adults over 65 are more likely to be vaccinated than other age groups in
} 


\section{Figure 2}

Results from the geospatial beta regression models estimating vaccination rates as a function of demographic, structural, political, and moral predictor variables

A

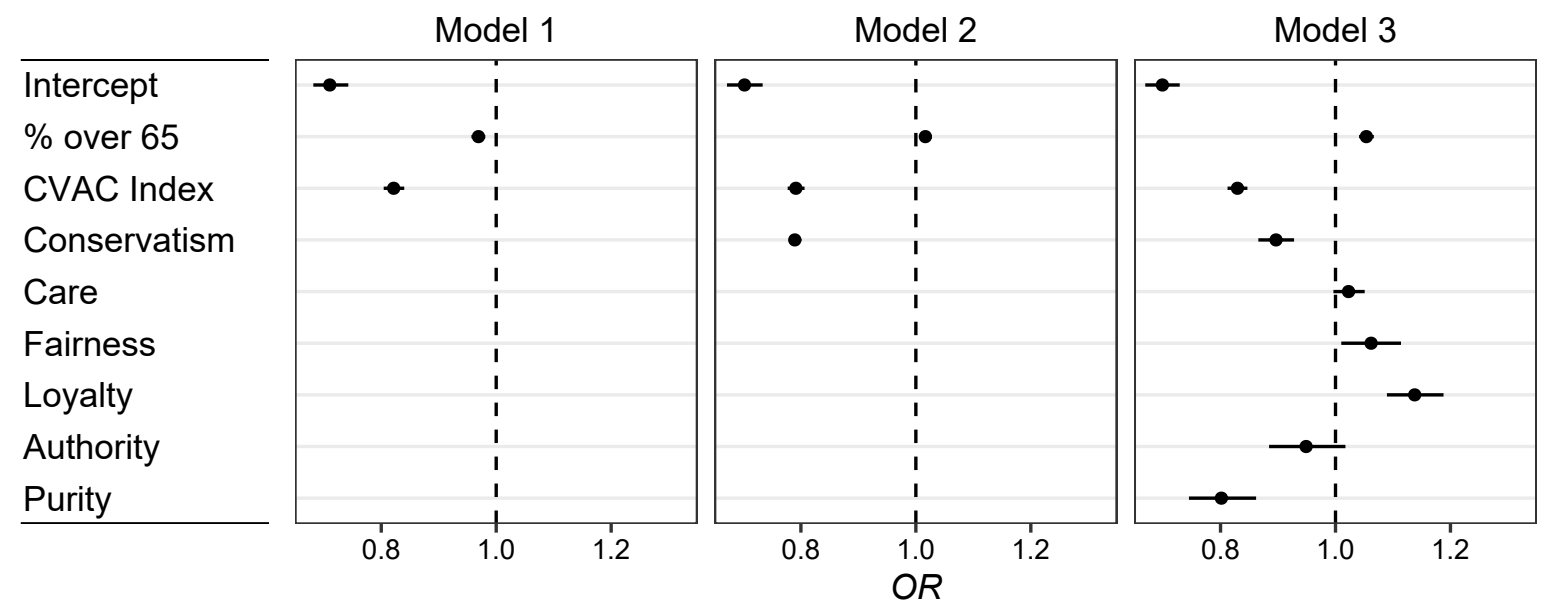

B

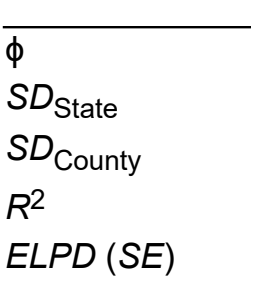

$\begin{array}{r}\text { Model } 1 \\ \hline 128.6[105.4,168.8] \\ 0.13[0.09,0.18] \\ 0.37[0.35,0.40] \\ 19 \%[16 \%, 22 \%] \\ -246.5(272.7) \\ \hline\end{array}$

Model 2

$129.7[108.9,171.1]$

$0.14[0.10,0.18]$

$0.27[0.25,0.30]$

$54 \%[52 \%, 56 \%]$ $1924.4(206.6)$
Model 3

$135.3[113.5,179.3]$

$0.13[0.10,0.18]$

$0.26[0.23,0.28]$

$64 \%[62 \%, 65 \%]$

$2483.4(195.8)$

Note. A Coefficients with $95 \%$ uncertainty intervals as odds ratios. B Dispersion parameter $(\phi)$ for the beta regression, standards deviations for the varying intercepts across states $\left(S D_{\text {State }}\right)$ and counties $\left(S D_{\text {County }}\right)$, and within-sample variance explained $\left(R^{2}\right)$ with $95 \%$ uncertainty intervals as well as the out-of-sample expected log predictive density $(E L P D)$ with its standard error $(S E)$.

explained $R^{2}=54 \%,[52 \%, 56 \%]$ of variance in vaccination rates and thus accounted for more within-sample variance than Model $1\left(\Delta R^{2}=35 \%\right.$, [31\%,39\%]). In the direction expected, a $1 S D$ increase in conservatism was associated with $O R=0.79,[0.78,0.80]$ times lower odds of vaccination. As Model 2 made more accurate out-of-sample predictions than

the United States (Center for Disease Control and Prevention, 2021). However, this association reverses in the expected direction in Models 2 and 3. This is an example of the ecological fallacy (Hehman et al., 2019; Robinson, 2009) since residents in counties with a greater proportion of residents over 65 also tend to be more conservative $(r=.17)$, lower in Care $(r=-.19)$ and Fairness $(r=-.26)$, and higher in Loyalty $(r=.20)$, Authority $(r=.14)$, and Purity $(r=.18)$. 
Model $1(\triangle E L P D=2170.9, S E=135.4)$ and this difference was $z=16.0$ times greater than its standards error, we conclude that political ideology predicts vaccination rates above and beyond structural and demographic barriers to vaccination in the United States.

Model 3, which included county-level endorsement of moral concerns as additional predictor variables, explained $R^{2}=64 \%,[62 \%, 65 \%]$ of variance in vaccination rates and thus accounted for more within-sample variance than Model $2\left(\Delta R^{2}=10 \%,[7 \%, 12 \%]\right)$. As shown in Figure 3, the predicted values for county-level vaccination rates based on Model 3 were highly consistent with the observed rates.

We found evidence that moral concerns about Fairness, Loyalty, and Purity, but not about Care or Authority, were associated with county-level vaccination rates. A $1 S D$ increase in Fairness was associated with $O R=1.06,[1.01,1.11]$ times higher odds of vaccination. Likewise, a $1 S D$ increase in Loyalty was associated with $O R=1.14,[1.09,1.19]$ times higher odds of vaccination. In contrast, a $1 S D$ increase in Purity was associated with $O R=0.80,[0.75,0.86]$ times lower odds of vaccination in line with our prediction. As Model 3 made more accurate out-of-sample predictions than Model $2(\triangle E L P D=559.0, S E=65.5)$ and this difference was $z=8.5$ times greater than its standard error, we conclude that moral concerns predict vaccination rates above and beyond political, demographic, and structural variables. ${ }^{12}$

To better understand the importance of each moral concern for predicting vaccination rates, we estimated five alternative versions of Model 3 that each included only a single moral concern as predictor variable (while controlling for demographic, structural, and political variables). We again found that Fairness $(O R=1.06,[1.03,1.09])$ was associated with higher odds of vaccination while Purity $(O R=0.87,[0.85,0.90])$ was

\footnotetext{
${ }^{12}$ A Model with fewer predictor variables can often make more accurate out-of-sample predictions than a model with more predictor variables. To find the model with the best out-of-sample prediction accuracy, we estimated 32 models that included all possible combinations of the five moral concerns (while controlling for demographic, structural, and political variables). We found that these models varied in their out-of-sample prediction accuracy and within-sample variance explained (Figure S3) and that a model with all moral foundations except authority outperformed a model with all moral foundations while all models with at least one moral foundation outperformed the model without any (Figure S4).
} 


\section{Figure 3}

Observed and predicted county-level vaccination rates

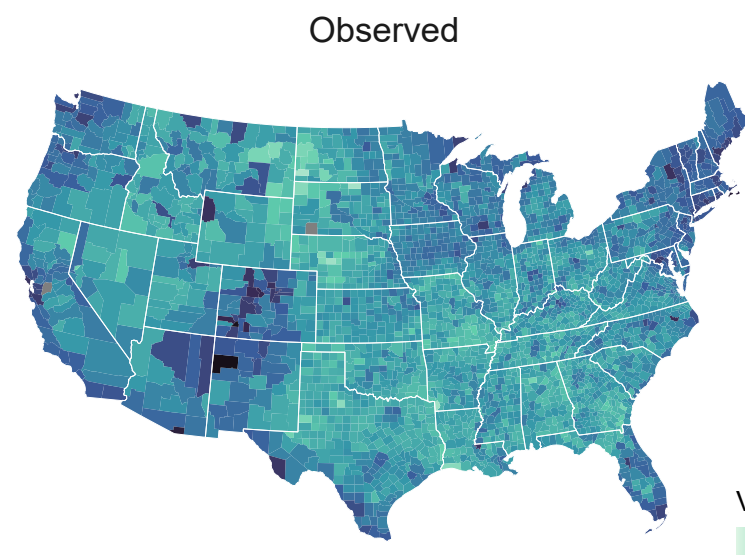

Model 2

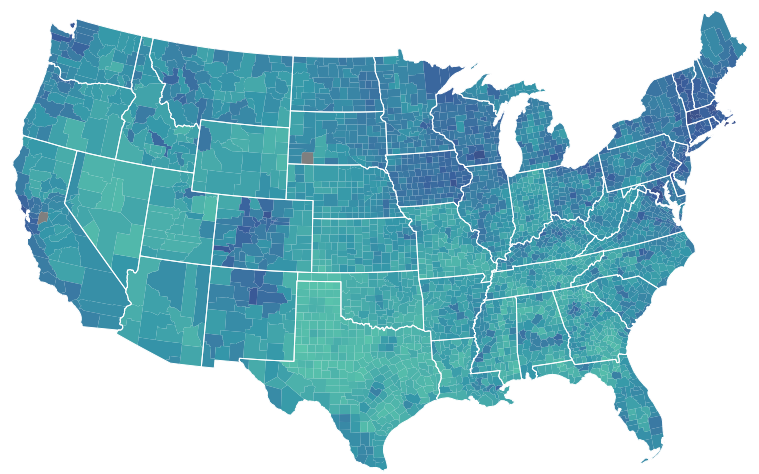

Model 1

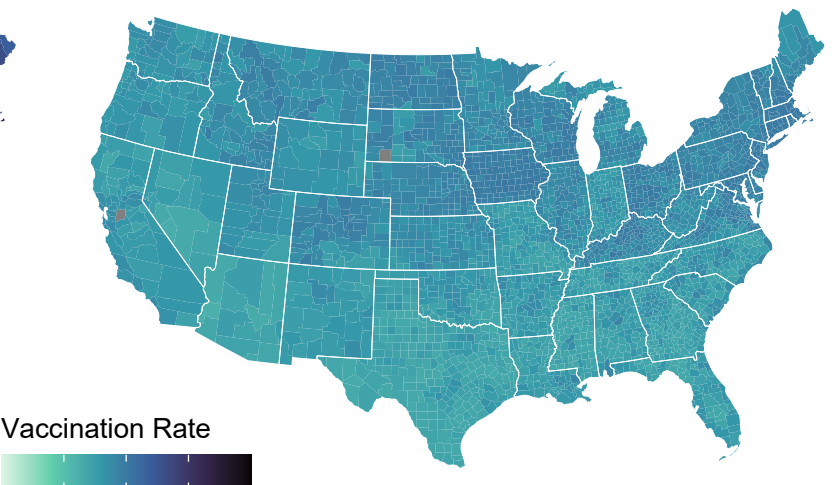

0.250 .500 .75

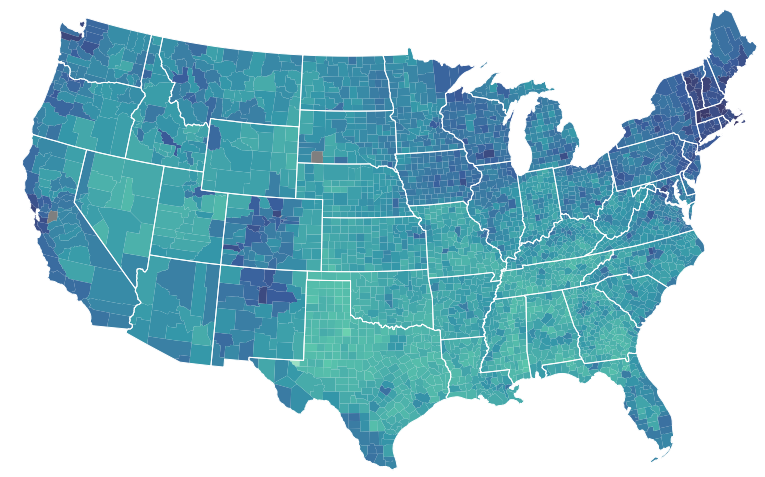

Note. Predicted vaccination rates reflect estimates from three geospatial beta regression models that included demographic and structural differences (Model 1), political differences (Model 2), and the five moral foundations (Model 3) as predictor variables of county-level vaccination rates. Predictions reflect only the non-spatial, fixed effects of each model.

associated with lower odds of vaccination (Figure S5). In addition, we found that Care $(O R=1.03,[1.02,1.05])$ was associated with higher odds of vaccination while Authority $(O R=0.92,[0.90,0.94])$ was associated with lower odds of vaccination. When not controlling for the other moral foundations, there was less evidence that Loyalty $(O R=0.98,[0.96,1.01])$ was associated with the odds of vaccination. 


\section{Robustness Checks}

Our main analyses controlled for, what we considered to be, the most plausible confounding variables - that is, structural and demographic barriers to vaccination - as well as for long-running voting tendencies to test whether county-level moral values would predict vaccination rates above and beyond differences in political ideology. That said, we recognize that the set of variables we chose to consider in our main analyses is only one of a multiverse of different sets of variables a reasonable researcher might have considered and that considering different sets of variables might have resulted in reaching different conclusions (Gelman \& Loken, 2014). To test how robust our conclusions were to making different analytic choices, we conducted a multiverse analysis (Steegen et al., 2016) in which we, first, defined a multiverse of all plausible combinations of analytic choices and, second, reran our analyses for each of those combinations.

Figures 4 and 5 show the results of our multiverse analysis. Each figure consists of three subfigures $(\mathrm{a}-\mathrm{c})$ which, in turn, consist of a lower and an upper section. Each vertical slice of a subfigure represents one analysis we ran. For example, the slice highlighted in red shows the result of Model 3 reported in the Main Analyses subsection-that is, a model that considers full vaccination rates as the outcome variable and controls for the proportion of over 65-year-olds, the CVAC index, and conservatism in the 20002020 presidential elections. The lower section of each subfigure uses lines to show, for each model we ran, which variables the model included besides the five moral foundations (not unlike a punch card). Specifically, we varied (in order of presentation) whether models considered full or partial vaccination rates as the outcome variable and whether models controlled for the proportion of over 65-year-olds; for the CVAC index, the CVAC subscales, or the CVAC subscales except historical undervaccination; for conservatism in the 2000-2020 presidential elections or in the 2020 presidential election; for rates of religious adherence; for the (log-transformed) population density; for the (log-transformed) median income and proportion of residents with a bachelors degree or higher; and for the proportions of White 
non-Latino, Black non-Latino, and Latino residents. In total, we thus estimated 768 models to test how robust our estimates of the relationships between county-level moral values and vaccination rates were across all combinations of analytic choices. The upper section of each subfigure $(\mathrm{a}-\mathrm{c})$ shows how $95 \%$ uncertainty intervals for the odds ratios for the relationships between county-level moral values and vaccination rates varied across the 768 models. Both parts of each subfigure show models ranked from the lowest (left) to the highest (right) odds ratio for a given moral foundation. If models that include a variable consistently rank higher than models that do not include that variable, we can conclude that the estimated effect sizes depend on including that variable.

We found that odds ratios for the relationships between county-level moral values and vaccination rates varied as a function of some analytic choices. Models that controlled for each county's racial composition estimated, on average, greater odds ratios for Authority $(O R=1.21 ; \Delta O R=0.25)$, Fairness $(O R=1.13 ; \Delta O R=0.10)$, and Purity $(O R=0.90 ; \Delta O R=0.05)$ but lower odds ratios for Loyalty $(O R=0.93 ; \Delta O R=-0.24)$ and Care $(O R=0.97 ; \Delta O R=-0.05)$ than models that did not include this control variable. Models that controlled for each county's conservatism in the 2020 presidential election estimated greater odds ratios for Purity $(O R=0.98 ; \Delta O R=0.16)$ and Loyalty $(O R=1.12 ; \Delta O R=0.10)$ but lower odds ratios for Fairness $(O R=0.99 ; \Delta O R=-0.14)$ and Authority $(O R=1.04 ; \Delta O R=-0.06)$ than models that did not include this control variable. In addition, models that controlled for each county's median income and education estimated, on average, greater odds ratios for Purity $(O R=0.92 ; \Delta O R=0.08)$. Other analytic choices, such as focusing on partial vaccination rates or controlling for religious adherence, did not, to the same extent, affect the estimated odds ratios $(|\Delta O R|<0.05)$. In summary, the multiverse analysis suggests that the relationships between moral values and vaccination rates might be sensitive to controlling for each county's racial composition and conservatism in the 2020 presidential election-but that 
Figure 4

Estimated odds ratios for the relationships of Care (a), Fairness (b), and Loyalty (c) with vaccination rates as a function of which other variables were included in the analysis

(a)
\% Full (Partial) Vaccination $\%$ over 65

CVAC Index

CVAC Subscales

CVAC Subscales (w/o Undervacc.)

Conservatism (2000-2020)

Conservatism (2020)

Religious Adherence

Population Density

Income/Education Race

(b)

\% Full (Partial) Vaccination
$\%$ over 65

CVAC Index

CVAC Subscales

CVAC Subscales (w/o Undervacc.)

Conservatism (2000-2020)

Conservatism (2020)

Religious Adherence

Population Density

Income/Education

Race

(c)

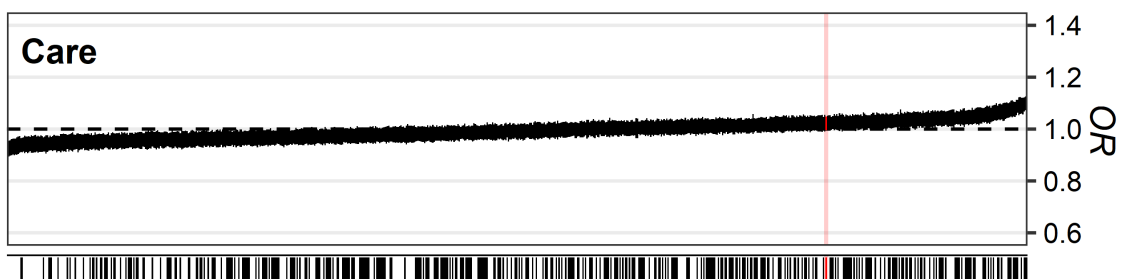

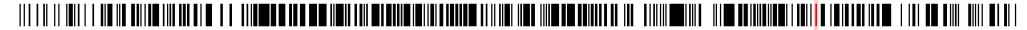

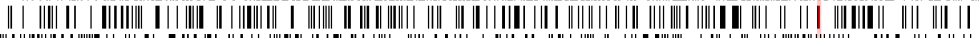

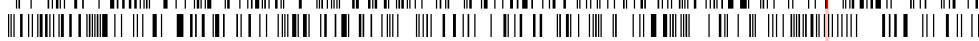

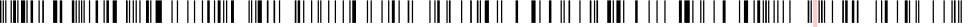

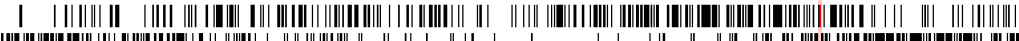

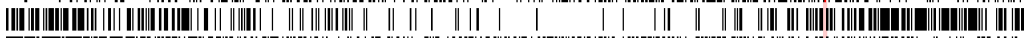

-

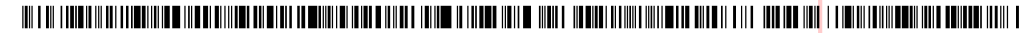

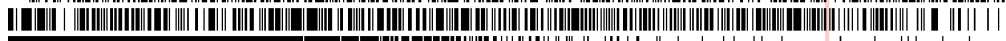

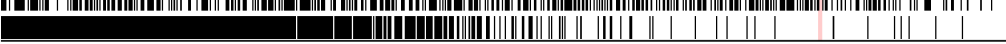

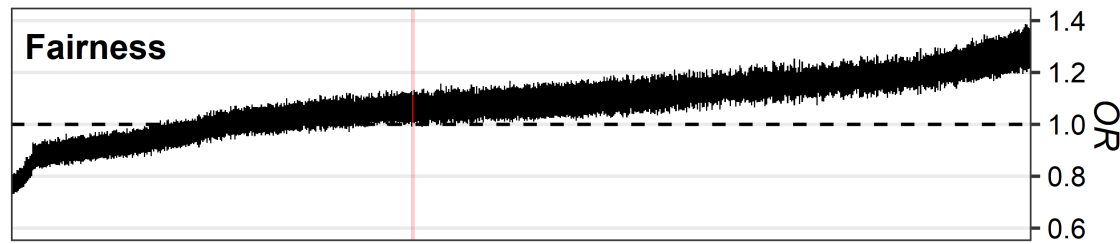

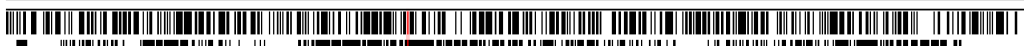

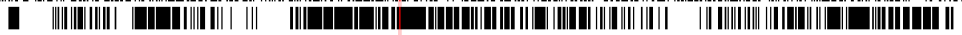

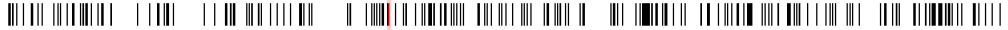

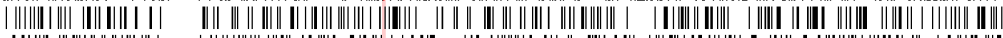

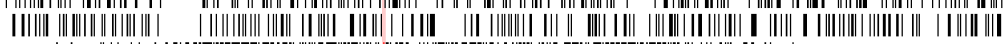

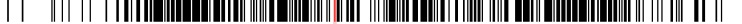

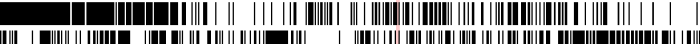

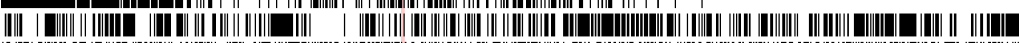

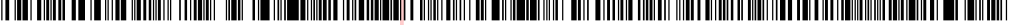

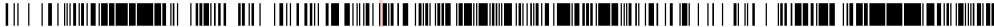
| |||||||||||| |||||||||||||||||||||||||||||||||||||||

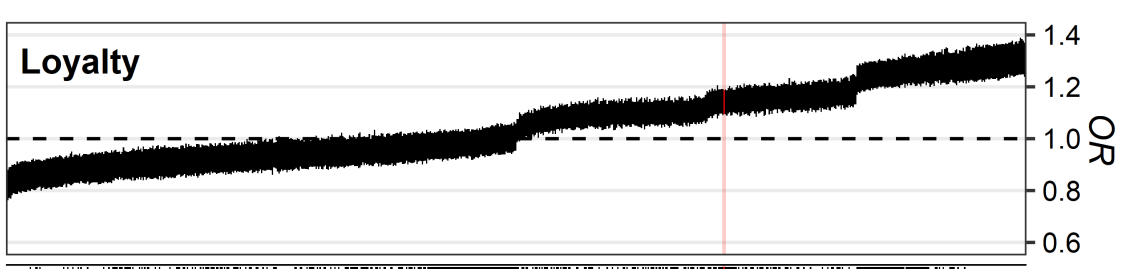

$\%$ Full (Partial) Vaccination

||| $|\||||||||||||||||||||||||||||||||||||||||||||||||||||||||||||$

||||||||||||||||||||||||||||||||||||||||||||||||||||||||

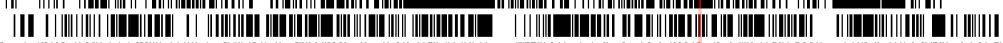

CVAC Index

CVAC Subscales

CVAC Subscales (w/o Undervacc.)

Conservatism (2000-2020)

Conservatism (2020)

Religious Adherence

Population Density

Income/Education

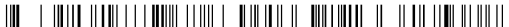

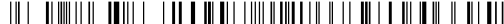

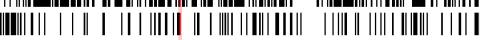

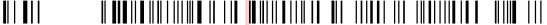

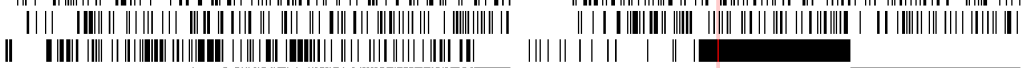

| | | |||||||||| | |||||||| ||||||||||||||||||||

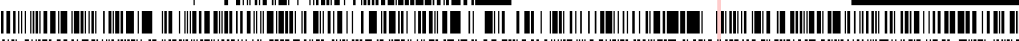

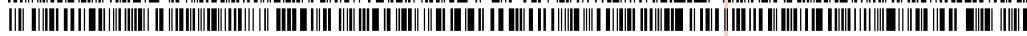

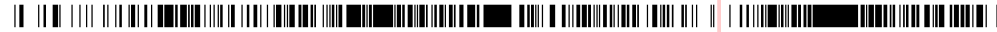

Note. Original model is highlighted in red. 


\section{Figure 5}

Estimated odds ratios for the relationships of Authority (a) and Purity (b) with vaccination rates and within-sample variance explained by all five moral foundations (c) as a function of which other variables were included in the analysis

(a)

(a)

\% Full (Partial) Vaccination
$\%$ over 65
CVAC Index
CVAC Subscales
CVAC Subscales (w/o Undervacc.)
Conservatism (2000-2020)
Conservatism (2020)
Religious Adherence
Population Density
Income/Education
Race

Race
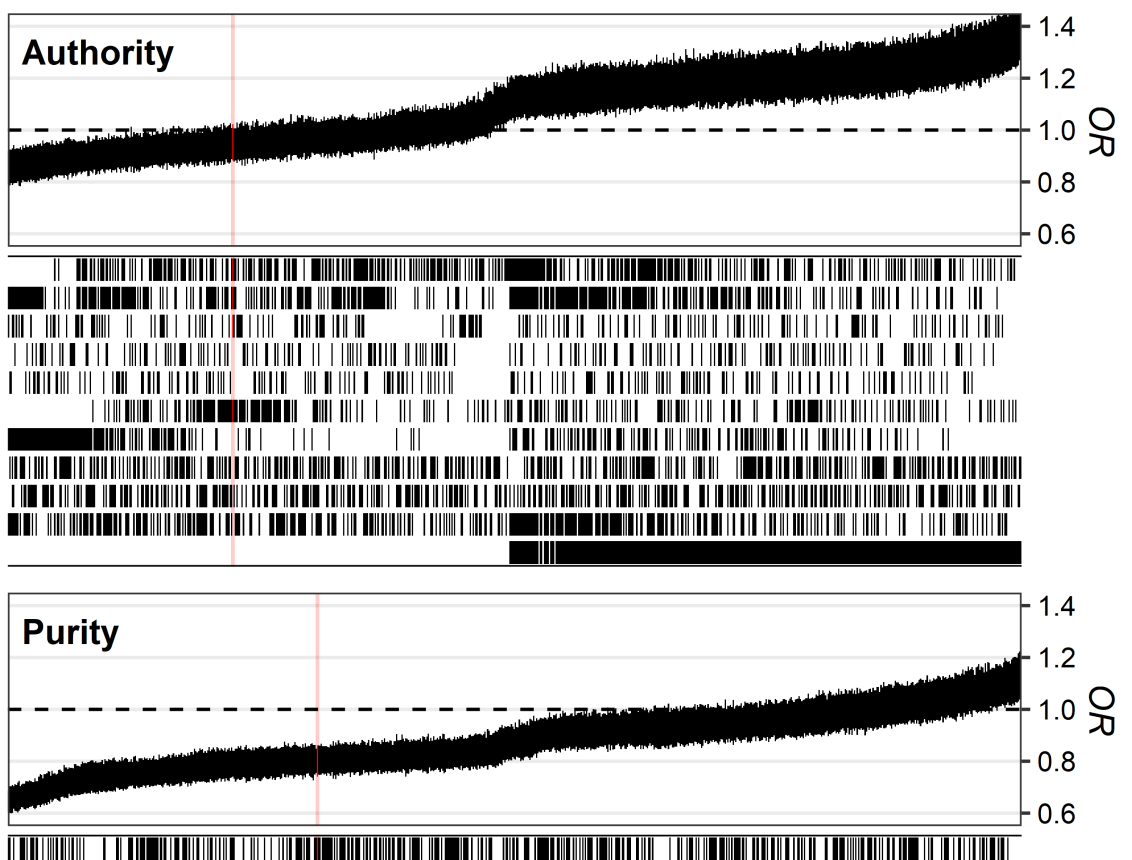

\% Full (Partial) Vaccination

$\%$ over 65

CVAC Index

CVAC Subscales

CVAC Subscales (w/o Undervacc.)

Conservatism (2000-2020)

Conservatism (2020)

Religious Adherence

Population Density

Income/Education

Race

(c)

\% Full (Partial) Vaccination
$\%$ over 65

CVAC Index

CVAC Subscales

CVAC Subscales (w/o Undervacc.)

Conservatism (2000-2020)

Conservatism (2020)

Religious Adherence

Population Density

Income/Education

Race

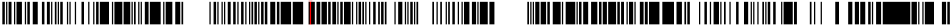

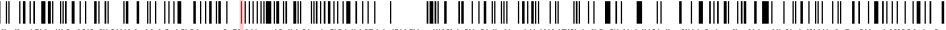

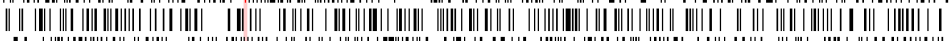

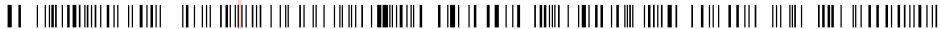

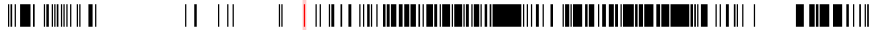
| |||||||||||||||||||||||||||||||||||||||||| $\mid$ | ||||||||||

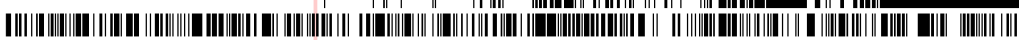

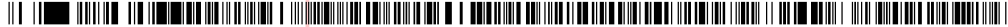

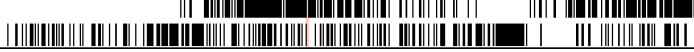

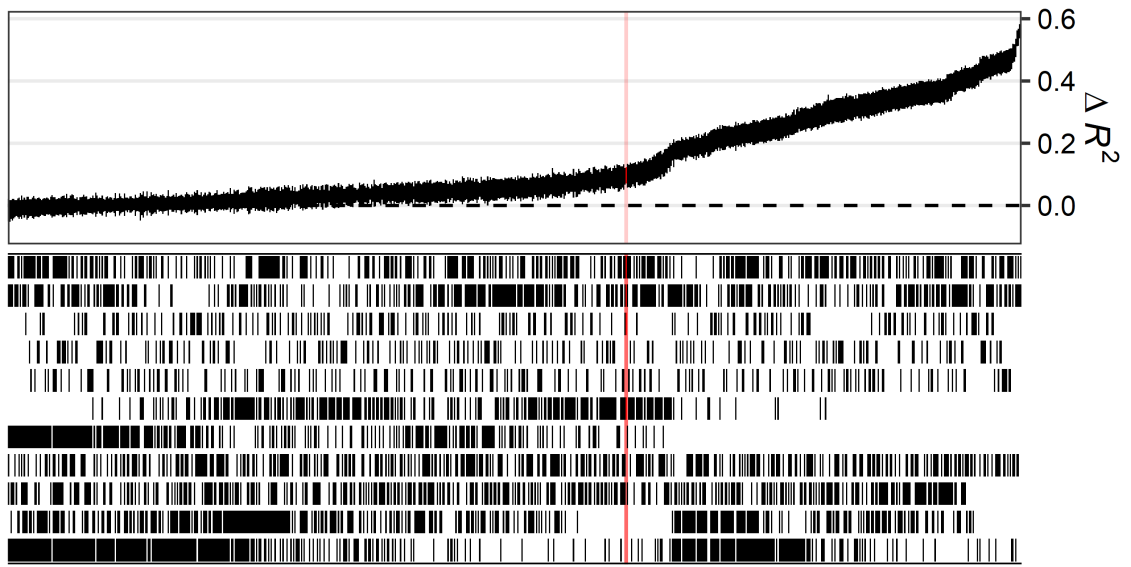

Note. $\Delta R^{2}$ is the estimated within-sample variance explained by the five moral foundations expressed as the difference in explained variance to a model that includes the same predictor variables but not the five moral foundations. Original model is highlighted in red. 
they are robust to most other analytic choices. ${ }^{13}$

Considering odds ratios for each moral foundation in isolation might, however, be misleading. For example, Loyalty was associated with higher odds of vaccination when not controlling for each county's racial composition $(O R=1.15,[1.04,1.33])$, as in the main analyses, whereas Authority was associated with higher odds of vaccination when controlling for each county's racial composition $(O R=1.20,[1.08,1.38])$. Figure 5 (c) shows the additional within-sample variance explained by all five moral foundations, expressed as the difference in variance explained by the control variables as well as the five moral foundations and the variance explained by the control variables alone $\left(\Delta R^{2}\right)$, as a function of which other variables were included in the model. Controlling for each county's conservatism in the 2020 presidential election $\left(\Delta R^{2}=.02\right.$ vs. $\left.\Delta R^{2}=.19\right)$ or in the 2000-2020 presidential elections ( $\Delta R^{2}=.06$ vs. $\left.\Delta R^{2}=.17\right)$-and, to a lesser extent, for each county's racial composition $\left(\Delta R^{2}=.10\right.$ vs. $\left.\Delta R^{2}=.17\right)$ or median income and education $\left(\Delta R^{2}=.11\right.$ vs. $\left.\Delta R^{2}=.16\right)$-reduced the additional within-sample variance explained by the five moral foundations. Other analytic choices did not reduce the estimated difference in the additional within-sample variance explained by county-level moral values. In summary, the multiverse analysis suggests that the importance of moral values in explaining county-level differences in vaccination rates is sensitive to controlling for differences in political ideology and racial composition - but robust to most other analytic choices. ${ }^{14}$

13 On average, a $1 S D$ increase in the proportion of Black, non-Latino $(O R=0.74,[0.64,0.83])$ and Latino/Hispanic $(O R=0.92,[0.87,0.97])$ residents, but not of White, non-Latino residents $(O R=0.86,[0.72,1.01])$, was associated with lower odds of vaccination. On average, a $1 S D$ increase in the 2020 Republican vote share was associated with lower odds of vaccination $(O R=0.65,[0.56,0.71])$.

${ }^{14}$ In the multiverse analysis, we focused on within-sample variance explained instead of out-of-sample prediction accuracy since running 10-fold cross-validations for 768 models would have been too computationally demanding. 


\section{Discussion}

The first COVID-19 vaccines received Emergency Use Authorization (EUA) in the United States in December of 2020. Despite the vaccines' demonstrated safety, efficacy, and abundant availability, as of September, 2021, many Americans refuse to get vaccinated. This refusal has had steep costs for the country: On September $9^{\text {th }}, 2021$, President Biden said "We've been patient. But our patience is wearing thin, and your refusal has cost all of us" before ordering sweeping federal mandates to combat the pandemic (Miller, 2021). While many universities and other institutions put vaccine mandates in place to provide a safe environment for their students and employees, in many states people can be granted exemptions on the basis of "philosophical", "religious", and "moral" reasons (Chuck, 2020). But what exactly are these moral concerns? In the present research we show that county-level moral values predict COVID-19 vaccination rates, even after adjusting for structural barriers, demographic characteristics, and political voting behavior. To our knowledge, this is the first study of the geospatial association between moral values and actual vaccination behavior.

Using Moral Foundations Theory's typology of moral concerns (Care, Fairness, Loyalty, Authority, and Purity), we found that counties with residents who prioritized Purity $(+1 S D)$ tended to have 0.80 times lower odds of vaccination. That is, our model predicted that if, in September 2021, a county had been average with respect to all other predictor variables, a 1 SD increase in Purity would, all else equal, have decreased vaccination rates by $-5 \%$. Several mechanisms might account for the relationship between Purity concerns at county-level and lower demand for COVID-19 vaccines. First, Purity concerns have been found to be associated with rejection of new things and novel experiences (Atari, Graham, et al., 2020; Lewis \& Bates, 2011), therefore, high concerns over keeping the human body's integrity and "naturalness" may be linked to avoiding these vaccines because they may be perceived as novel, contaminated, and unnatural (Scott et al., 2016). This is consistent with some of the reasons Americans in conservative-leaning 
counties mention when asked about their refusal. For example, individuals may be worried that the vaccines are too new (e.g., "I'm really cautious about what goes into my body"; Bosman et al., 2021). Second, it might be possible that individuals who have heightened Purity concerns may hold less favorable attitudes toward science as well as stronger conspiracist beliefs which in turn may discourage them to voluntarily get the COVID-19 vaccine (Pivetti et al., 2021).

In addition, we found that, after accounting for their residents' political behavior, counties with residents who prioritized Loyalty or Fairness $(+1 S D)$ tended to have, respectively, 1.13 and 1.07 times higher odds of vaccination. That is, our model predicted that if, in September 2021, a county had been average with respect to all other predictor variables, a 1 SD increase in Fairness or Loyalty would, all else equal, have increased vaccination rates by $+2 \%$ and $+3 \%$, respectively. That Fairness concerns predict higher demand for COVID-19 vaccinations is unsurprising: Reporting on the pandemic has repeatedly stressed the importance of protecting vulnerable groups (e.g., older or immunocompromised people) from infection and highlighted the disparate impact of COVID-19 on disadvantaged communities. That Loyalty concerns predict higher demand for COVID-19 vaccinations is, in some ways, surprising: After all, Loyalty concerns are consistently associated with political conservatism (Graham et al., 2009; Kivikangas et al., 2021) which, in the United States, has been consistently associated with resistance to vaccination. Getting vaccinated, however, can also be understood as a patriotic act to protect one's ingroup and relatives from infection - an idea that seems to have intuitive appeal as political leaders have used it to urge U.S. citizens to get vaccinated (e.g., "If you haven't gotten vaccinated, please do it now. It's the most patriotic thing you can do," Biden, 2021a; "Get vaccinated now ... its your patriotic duty," Biden, 2021b). That said, this finding should be interpreted with some caution as Loyalty was only associated with (higher) vaccination rates when controlling for the four other moral foundations - suggesting that Loyalty to one's country is, on its own, not enough to 
motivate vaccination behavior. This does not, however, rule out that connecting vaccination to Loyalty can be an effective strategy to convince vaccine-hesitant citizens to get vaccinated.

While generally caution should be exercised when inferring policy insights from behavioral and psychological findings (IJzerman et al., 2020), the present geospatial relation between moral values and vaccination behavior may have important implications for health communication and large-scale interventions. For example, in high-Purity regions, health communication can be framed in a way that is consistent with that county's Purity concerns among other values, a technique called "moral reframing", a communication tool that can work across the political divide (Feinberg \& Willer, 2019). An interesting case of moral reframing can be seen Utah; after tailing in national vaccination rates, the Mormon leaders started referring to the COVID-19 vaccine as a "literal godsend" and an answer to their prayers (Walch, 2021), which resulted in a boost in vaccination rates (Alberty, 2021) ${ }^{15}$. Similar strategies can be used to frame the vaccine as a protective shield guarding against the disgusting contaminating disease.

Likewise, in vaccine-hesitant conservative regions, health communication can appeal to residents' Loyalty concerns by framing vaccination as a patriotic duty to protect fellow citizens. Morally reframed messages can be particularly influential because the recipients perceive a "match" between their own moral convictions and the argument in favor of the other side's policy position. Moral convictions are immutable aspects of one's identity (Skitka et al., 2021; Strohminger \& Nichols, 2014). Since moral convictions are held so strongly, arguments that appeal to them are difficult to discount, even when used to argue for a position one would typically oppose. Indeed, some moral reframing techniques have already been shown to be effective in health communication during the COVID-19 pandemic (Kaplan et al., 2021). These intervention-related insights may be incorporated with prior effective communication strategies (e.g., Jung \& Albarracn, 2021) to encourage

\footnotetext{
${ }^{15}$ We would like to thank Fritz Götz for this example.
} 
people to get vaccinated.

While our results are the first to characterize the role of county-level moral values in predicting the regional uptake of COVID-19 vaccines, and to examine real-world behavior instead of self-reported vaccine hesitancy or intentions, several limitations are worth noting. First, caution should be applied when interpreting our geospatial findings, that is, just because a county that is high in Purity is more likely to have a low vaccination rate does not necessarily imply that this association is driven by residents high in Purity refusing to get vaccinated (the ecological fallacy; see, for example, Hehman et al., 2019). We argue, however, that an individual-level interpretation of county-level associations between moral values and vaccination behavior is indeed plausible as our findings align with findings of prior individual-level studies (Amin et al., 2017; Pivetti et al., 2021) and as our analyses controlled for a broad range of alternative explanations for these county-level associations. Related to this issue, caution also should be applied when generalizing our findings to other levels of spatial aggregation, that is, just because counties higher in Purity tend to have low vaccination rates does not imply that the same association holds across more granular (e.g., towns or neighborhoods) or less granular (e.g., states) levels of analysis. This potential lack of generalizability across spatial units has been termed the Modifiable Aerial Unit Problem (Manley, 2013). Previous psychological research using geospatial analysis have been able to replicate the found construct-outcome associations across different spatial layers (e.g. Ebert, Götz, et al., 2021), but this is not always been the case (Ebert, Gebauer, et al., 2021) ${ }^{16}$.

Second, our results may not generalize to other countries. A crucial context which made the testing of our predictions possible was the fact that vaccines have been abundantly available in the United States. This certainly is not the case in many other countries at the moment; however, future work is encouraged to replicate these regional findings when vaccines become largely available for the general public across countries.

16 We would like to thank Fritz Götz for pointing out this potential limitation. 
Third, our study was not prospective in nature, hence these relationships are subject to change in the future; however, given that vaccines have been conveniently and freely available for several months, that is unlikely. Fourth, we acknowledge that our MFQ data, collected between 2012 to 2018, is temporally out of sequence with the CVAC and vaccination data (both collected in 2021). We note that there is evidence that individual differences in adherence to moral concerns (Graham et al., 2011) as well as regional differences in other psychological characteristics (Elleman et al., 2018) are relatively stable over time. Nonetheless, future research should further investigate the temporal dependency between moral concerns and moral behavior at the aggregate level.

A final limitation is that our findings are, to some extent, specific to the analytic choices we made. Our multiverse analysis suggested that controlling for each county's racial composition and for how its residents voted in the 2020 presidential election would have diminished the importance of county-level moral values for explaining differences in vaccination rates. Controlling for a variable tends to assume that the control variable affects both the predictor and outcome variable and, if not controlled for, confounds the relationship between the two - that is, it assumes a specific causal model for the relationships between the control, predictor, and outcome variables (Rohrer, 2018). However, this assumption is not always the most plausible causal model. For example, while it is likely that support for Donald J. Trump in the 2020 presidential election is a proximal cause of lower vaccination rates, it is not plausible that it also explains differences in moral values based on data from 2012 to 2018. As such, the voting behavior in the 2020 presidential election might be a mediator, rather than a confounder, in the relationships between moral values, political ideology, and vaccination behavior. ${ }^{17}$ In contrast, our multiverse analysis showed that our findings are robust to a broad range of other analytic choices, such as controlling for religious adherence or using partial vaccination rates as the

\footnotetext{
17 Relatedly, we calculated our index of political ideology based on a longer time span (2000-2020) to
} capture long-running voting tendencies instead of short-term political preferences. 
relevant outcome variables.

\section{Conclusion}

Combining county-level estimates of moral values and of real-world vaccination behavior, we demonstrated that moral values add to our understanding and prediction of vaccination rates during the COVID-19 pandemic at a regional level of analysis. Our

findings showed that higher Purity concerns about bodily and spiritual contamination, lower Loyalty concerns about ingroup cooperation, and lower Fairness concerns about equality and proportionality are associated with lower rates of COVID-19 vaccination, even after controlling for structural barriers, demographic make-up, and political voting behavior across U.S. counties. 


\section{References}

Agarwal, R., Dugas, M., Ramaprasad, J., Luo, J., Li, G., \& Gao, G. G. (2021).

Socioeconomic privilege and political ideology are associated with racial disparity in COVID-19 vaccination. Proceedings of the National Academy of Sciences, 118(33).

Alberty, E. (2021). Here's what happened to utah's vaccination rates after lds church leaders told members to get their shots. The Salt Lake Tribune. Retrieved August 26, 2021, from https://www.sltrib.com/news/2021/08/26/heres-what-happened-utahs/

Amin, A. B., Bednarczyk, R. A., Ray, C. E., Melchiori, K. J., Graham, J., Huntsinger, J. R., \& Omer, S. B. (2017). Association of moral values with vaccine hesitancy. Nature Human Behaviour, 1(12), 873-880.

Atari, M., Graham, J., \& Dehghani, M. (2020). Foundations of morality in Iran. Evolution and Human Behavior, 41(5), 367-384.

Atari, M., Haidt, J., Graham, J., Koleva, S., Stevens, S. T., \& Dehghani, M. (2022). Morality beyond the weird: How the nomological network of morality varies across cultures.

Atari, M., Mostafazadeh Davani, A., \& Dehghani, M. (2020). Body maps of moral concerns. Psychological science, 31(2), 160-169.

Baumeister, R. F., Vohs, K. D., \& Funder, D. C. (2007). Psychology as the science of self-reports and finger movements: Whatever happened to actual behavior? Perspectives on psychological science, 2(4), 396-403.

Baumgaertner, B., Carlisle, J. E., \& Justwan, F. (2018). The influence of political ideology and trust on willingness to vaccinate. PloS one, 13(1), e0191728.

Betsch, C., \& Böhm, R. (2018). Moral values do not affect prosocial vaccination. Nature human behaviour, 2(12), 881-882.

Biden, J. R. [@POTUS]. (2021a, July 4). If you haven't gotten vaccinated, please do it now. It's the most patriotic thing you can do. [Tweet]. Twitter. Retrieved September 13, 2021, from https://twitter.com/potus/status/1411831187178467329 
Biden, J. R. [@POTUS]. (2021b, December 21). To those people who have not been vaccinated: You have an obligation to yourself, your family, and your country. Get vaccinated now. Its free. Its convenient. It saves lives. And its your patriotic duty. [Tweet]. Twitter. Retrieved January 5, 2022, from https://twitter.com/POTUS/status/1473421587953168384

Bosman, J., Hoffman, J., Sanger-Katz, M., \& Arango, T. (2021). Who are the unvaccinated in America? There's no one answer. The New York Times. Retrieved September 10, 2021, from https://www.nytimes.com/2021/07/31/us/virus-unvaccinated-americans.html

Bürkner, P.-C. (2017). Brms: An R package for Bayesian multilevel models using Stan. Journal of Statistical Software, 80(1). https://doi.org/10.18637/jss.v080.i01

Bürkner, P.-C. (2018). Advanced Bayesian multilevel modeling with the R package brms. The R Journal, 10(1), 395-411. https://doi.org/10.32614/RJ-2018-017

Center for Disease Control and Prevention. (2021). Demographic trends of people receiving COVID-19 vaccinations in the United States [Data Set]. Retrieved December 9, 2021, from https://covid.cdc.gov/covid-data-tracker/\#vaccination-demographics-trends

Chuck, E. (2020). Many parents are hesitant to give their kids a Covid-19 vaccine. What if schools require it? NBC News. Retrieved September 9, 2021, from https://www.nbcnews.com/news/us-news/many-parents-are-hesitant-give-theirkids-covid-19-vaccine-n1240741

Cushman, F. (2013). Action, outcome, and value: A dual-system framework for morality. Personality and social psychology review, 17(3), 273-292.

De Figueiredo, A., Simas, C., Karafillakis, E., Paterson, P., \& Larson, H. J. (2020). Mapping global trends in vaccine confidence and investigating barriers to vaccine uptake: A large-scale retrospective temporal modelling study. The Lancet, $396(10255), 898-908$. 
Dubé, E., Laberge, C., Guay, M., Bramadat, P., Roy, R., \& Bettinger, J. A. (2013). Vaccine hesitancy: An overview. Human Vaccines $\mathcal{E}$ Immunotherapeutics, 9(8), 1763-1773.

Ebert, T., Gebauer, J. E., Brenner, T., Bleidorn, W., Gosling, S. D., Potter, J., \& Rentfrow, P. J. (2021). Are regional differences in psychological characteristics and their correlates robust? applying spatial-analysis techniques to examine regional variation in personality. Perspectives on Psychological Science, 1745691621998326.

Ebert, T., Götz, F. M., Gladstone, J. J., Müller, S. R., \& Matz, S. C. (2021). Spending reflects not only who we are but also who we are around: The joint effects of individual and geographic personality on consumption. Journal of Personality and Social Psychology, $121(2), 378$.

Elleman, L. G., Condon, D. M., Russin, S. E., \& Revelle, W. (2018). The personality of U.S. states: Stability from 1999 to 2015. Journal of Research in Personality, 72, 64-72. https://doi.org/10.1016/j.jrp.2016.06.022

Feinberg, M., \& Willer, R. (2019). Moral reframing: A technique for effective and persuasive communication across political divides. Social and Personality Psychology Compass, 13(12), e12501.

Ferrari, S., \& Cribari-Neto, F. (2004). Beta regression for modelling rates and proportions. Journal of Applied Statistics, 31(7), 799-815. https://doi.org/10.1080/0266476042000214501

Fridman, A., Gershon, R., \& Gneezy, A. (2021). COVID-19 and vaccine hesitancy: A longitudinal study. PloS one, 16(4). https://doi.org/10.1371/journal.pone.0250123

Gelman, A., Goodrich, B., Gabry, J., \& Vehtari, A. (2019). R-squared for Bayesian regression models. The American Statistician, 73(3), 307-309. https://doi.org/10.1080/00031305.2018.1549100

Gelman, A., \& Loken, E. (2014). The statistical crisis in science. American Scientist, 102(6), 460-465. https://doi.org/10.1511/2014.111.460 
Graham, J. (2013). Beyond economic games: A mutualistic approach to the rest of moral life. Behavioral and Brain Sciences, 36(1), 91.

Graham, J., \& Haidt, J. (2012). Sacred values and evil adversaries: A moral foundations approach. In M. Mikulincer \& P. R. Shaver (Eds.), The social psychology of morality: Exploring the causes of good and evil (pp. 11-31). American Psychological Association.

Graham, J., Haidt, J., Koleva, S., Motyl, M., Iyer, R., Wojcik, S. P., \& Ditto, P. H. (2013). Moral foundations theory: The pragmatic validity of moral pluralism. Advances in experimental social psychology, 47, 55-130.

Graham, J., Haidt, J., \& Nosek, B. A. (2009). Liberals and conservatives rely on different sets of moral foundations. Journal of Personality and Social Psychology, 96(5), 1029-1046.

Graham, J., Nosek, B. A., Haidt, J., Iyer, R., Koleva, S., \& Ditto, P. H. (2011). Mapping the moral domain. Journal of personality and social psychology, 101(2), 366-385.

Grammich, C., Hadaway, K., Houseal, R., Jones, D. E., Krindatch, A., Stanley, R., \& Taylor, R. H. (2010). U.S. religion census religious congregations and membership study, 2010 [Data Set]. https://doi.org/10.17605/OSF.IO/QUN29

Gray, K., \& Wegner, D. M. (2009). Moral typecasting: Divergent perceptions of moral agents and moral patients. Journal of personality and social psychology, 96(3), 505.

Gursoy, D., Ekinci, Y., Can, A. S., \& Murray, J. C. (2022). Effectiveness of message framing in changing covid-19 vaccination intentions: Moderating role of travel desire. Tourism Management, 90, 104468.

Haidt, J. (2001). The emotional dog and its rational tail: A social intuitionist approach to moral judgment. Psychological review, 108(4), 814-834.

Haidt, J., \& Joseph, C. (2004). Intuitive ethics: How innately prepared intuitions generate culturally variable virtues. Daedalus, 133(4), 55-66. 
Hanretty, C., Lauderdale, B. E., \& Vivyan, N. (2016). Comparing strategies for estimating constituency opinion from national survey samples. Political Science Research and Methods, 6(3), 571-591.

Hehman, E., Calanchini, J., Flake, J. K., \& Leitner, J. B. (2019). Establishing construct validity evidence for regional measures of explicit and implicit racial bias. Journal of experimental psychology: General, 148(6), 1022.

Hoover, J., Atari, M., Davani, A. M., Kennedy, B., Portillo-Wightman, G., Yeh, L., \& Dehghani, M. (2021). Investigating the role of group-based morality in extreme behavioral expressions of prejudice. Nature Communications, 12(1), 1-13.

Hoover, J., \& Dehghani, M. (2019). The big, the bad, and the ugly: Geographic estimation with flawed psychological data. Psychological Methods, 25(4), 412-429. https://doi.org/10.1037/met0000240

Hornsey, M. J., Finlayson, M., Chatwood, G., \& Begeny, C. T. (2020). Donald Trump and vaccination: The effect of political identity, conspiracist ideation and presidential tweets on vaccine hesitancy. Journal of Experimental Social Psychology, 88, 103947. https://doi.org/10.1016/j.jesp.2019.103947

Hornsey, M. J., Harris, E. A., \& Fielding, K. S. (2018). The psychological roots of anti-vaccination attitudes: A 24-nation investigation. Health psychology, 37(4), 307.

IJzerman, H., Lewis, N. A., Przybylski, A. K., Weinstein, N., DeBruine, L., Ritchie, S. J., Vazire, S., Forscher, P. S., Morey, R. D., Ivory, J. D., \& Anvari, F. (2020). Use caution when applying behavioural science to policy. Nature Human Behaviour, 4(11), 1092-1094.

Ivory, D., Leatherby, L., \& Gebeloff, R. (2021). Least vaccinated us counties have something in common: Trump voters. The New York Times. Retrieved September 9, 2021, from https://www.nytimes.com/interactive/2021/04/17/us/vaccine-hesitancypolitics.html 
Iyer, R., Koleva, S., Graham, J., Ditto, P., \& Haidt, J. (2012). Understanding libertarian morality: The psychological dispositions of self-identified libertarians. PLoS ONE, e42366.

Janoff-Bulman, R., \& Carnes, N. C. (2013). Surveying the moral landscape: Moral motives and group-based moralities. Personality and Social Psychology Review, 17(3), 219-236.

Jokela, M., Bleidorn, W., Lamb, M. E., Gosling, S. D., \& Rentfrow, P. J. (2015). Geographically varying associations between personality and life satisfaction in the london metropolitan area. Proceedings of the National Academy of Sciences, 112(3), $725-730$.

Jung, H., \& Albarracn, D. (2021). Concerns for others increases the likelihood of vaccination against influenza and COVID-19 more in sparsely rather than densely populated areas. Proceedings of the National Academy of Sciences, 118(1), e2007538118.

Kaplan, J., Vaccaro, A., Henning, M., \& Christov-Moore, L. (2021). Moral reframing of messages about mask-wearing during the COVID-19 pandemic. https://doi.org/10.31234/osf.io/gfa5r

Killgore, W. D., Cloonan, S. A., Taylor, E. C., \& Dailey, N. S. (2021). The COVID-19 vaccine is here-Now who is willing to get it? Vaccines, 9(4), 339.

Kivikangas, J. M., Fernández-Castilla, B., Järvelä, S., Ravaja, N., \& Lönnqvist, J.-E. (2021). Moral foundations and political orientation: Systematic review and meta-analysis. Psychological Bulletin, 147(1), 55-94. https://doi.org/10.1037/bul0000308

Koinig, I. (2021). On the influence of message/audience specifics and message appeal type on message empowerment: The austrian case of covid-19 health risk messages. Health Communication, 1-12. 
Latkin, C. A., Dayton, L., Yi, G., Colon, B., \& Kong, X. (2021). Mask usage, social distancing, racial, and gender correlates of COVID-19 vaccine intentions among adults in the US. PloS one, 16(2). https://doi.org/10.1371/journal.pone.0246970

Leemann, L., \& Wasserfallen, F. (2017). Extending the use and prediction precision of subnational public opinion estimation: Extending use and precision of MrP. American Journal of Political Science, 61(4), 1003-1022.

Lewis, G. J., \& Bates, T. C. (2011). From left to right: How the personality system allows basic traits to influence politics via characteristic moral adaptations. British Journal of Psychology, 102(3), 546-558.

MacDonald, N. E. (2015). Vaccine hesitancy: Definition, scope and determinants. Vaccine, $33(34), 4161-4164$.

Mandavilli, A. (2021). The delta variant is the symptom of a bigger threat: Vaccine refusal. Retrieved September 11, 2021, from https://www.nytimes.com/2021/07/25/health/coronavirus-vaccine-refusal.html

Manley, D. J. (2013). Scale, aggregation, and the modifiable areal unit problem. Handbook of regional science. Springer Verlag.

Miller, Z. (2021). Sweeping new vaccine mandates for 100 million Americans. The Associated Press. Retrieved September 9, 2021, from https://apnews.com/article/joe-biden-business-health-coronavirus-pandemicexecutive-branch-18fb12993f05be13bf760946a6fb89be

Mishra, A., Sutermaster, S., Smittenaar, P., Stewart, N., \& Sgaier, S. (2021). COVID-19 vaccine coverage index: Identifying barriers to COVID-19 vaccine uptake across US counties. medRxiv. https://doi.org/10.1101/2021.06.17.21259116

MIT Election Data and Science Lab. (2018). County presidential election returns 2000-2016. Harvard Dataverse. https://doi.org/10.7910/DVN/VOQCHQ

Morris, M., Wheeler-Martin, K., Simpson, D., Mooney, S. J., Gelman, A., \& DiMaggio, C. (2019). Bayesian hierarchical spatial models: Implementing the Besag York Mollié 
model in Stan. Spatial and Spatio-temporal Epidemiology, 31, 100301. https://doi.org/10.1016/j.sste.2019.100301

Naterattner. (2021). Covid vaccinations are slowing in the U.S. as supply outstrips demand. How states are targeting who's left. Retrieved September 12, 2021, from https://www.cnbc.com/2021/04/30/covid-vaccinations-in-us-are-slowing-as-supplyoutstrips-demand.html

Nkengasong, J. N., Ndembi, N., Tshangela, A., \& Raji, T. (2020). COVID-19 vaccines: How to ensure Africa has access. Nature, 586(7828), 197-199.

Nowok, B., Raab, G. M., \& Dibben, C. (2016). synthpop: Bespoke creation of synthetic data in R. Journal of Statistical Software, $74(11), 1-26$. https://doi.org/10.18637/jss.v074.i11

Park, D. K., Gelman, A., \& Bafumi, J. (2004). Bayesian multilevel estimation with poststratification: State-level estimates from national polls. Political Analysis, $12(4), 375-385$.

Peters, H., Götz, F. M., Ebert, T., Müller, S., Rentfrow, J., Gosling, S. D., Obschonka, M., Ames, D., Potter, J., \& Matz, S. (2020). Regional personality differences predict variation in COVID-19 infections and social distancing behavior (preprint). PsyArXiv. https://doi.org/10.31234/osf.io/sqh98

Pivetti, M., Melotti, G., Bonomo, M., \& Hakoköngäs, E. (2021). Conspiracy beliefs and acceptance of COVID-Vaccine: An exploratory study in Italy. Social Sciences, $10(3), 108$.

Rai, T. S., \& Fiske, A. P. (2011). Moral psychology is relationship regulation: Moral motives for unity, hierarchy, equality, and proportionality. Psychological review, $118(1), 57$.

Reinhart, R. (2020). Fewer in US continue to see vaccines as important. Gallup, January, 14. Retrieved September 9, 2021, from https://news.gallup.com/poll/276929/fewer-continue-vaccines-important.aspx 
Rentfrow, P. J., Gosling, S. D., \& Potter, J. (2008). A theory of the emergence, persistence, and expression of geographic variation in psychological characteristics. Perspectives on Psychological Science, 3(5), 339-369. https://doi.org/10.1111/j.1745-6924.2008.00084.x

Rentfrow, P. J. (2010). Statewide differences in personality: Toward a psychological geography of the United States. American Psychologist, 65(6), 548-558. https://doi.org/10.1037/a0018194

Reuben, R., Aitken, D., Freedman, J. L., \& Einstein, G. (2020). Mistrust of the medical profession and higher disgust sensitivity predict parental vaccine hesitancy. PLoS One, 15(9), e0237755.

Riebler, A., Sørbye, S. H., Simpson, D., \& Rue, H. (2016). An intuitive Bayesian spatial model for disease mapping that accounts for scaling. Statistical Methods in Medical Research, 25(4), 1145-1165. https://doi.org/10.1177/0962280216660421

Robinson, W. S. (2009). Ecological correlations and the behavior of individuals. International journal of epidemiology, 38(2), 337-341.

Rohrer, J. M. (2018). Thinking clearly about correlations and causation: Graphical causal models for observational data. Advances in Methods and Practices in Psychological Science, 1(1), 27-42. https://doi.org/10.1177/2515245917745629

Schoch-Spana, M., Brunson, E. K., Long, R., Ruth, A., Ravi, S. J., Trotochaud, M., Borio, L., Brewer, J., Buccina, J., Connell, N., Hall, L. L., Kass, N., Kirkland, A., Koonin, L., Larson, H., Lu, B. F., Omer, S. B., Orenstein, W. A., Poland, G. A., ... White, A. (2020). The publics role in COVID-19 vaccination: Human-centered recommendations to enhance pandemic vaccine awareness, access, and acceptance in the United States. Vaccine.

Schwartz, S. H. (1992). Universals in the content and structure of values: Theoretical advances and empirical tests in 20 countries. In M. P. Zanna (Ed.), Advances in experimental social psychology (pp. 1-65). Academic Press. 
Scott, S. E., Inbar, Y., \& Rozin, P. (2016). Evidence for absolute moral opposition to genetically modified food in the United States. Perspectives on Psychological Science, $11(3), 315-324$.

Shimabukuro, T. T., Kim, S. Y., Myers, T. R., Moro, P. L., Oduyebo, T., Panagiotakopoulos, L., Marquez, P. L., Olson, C. K., Liu, R., Chang, K. T., Ellington, S. R., Burkel, V. K., Smoots, A. N., Green, C. J., Licata, C., Zhang, B. C., Alimchandani, M., Mba-Jonas, A., Martin, S. W., ... Meaney-Delman, D. M. (2021). Preliminary findings of mRNA Covid-19 vaccine safety in pregnant persons. New England Journal of Medicine, 384(24), 2273-2282.

Skitka, L. J., Hanson, B. E., Morgan, G. S., \& Wisneski, D. C. (2021). The psychology of moral conviction. Annual Review of Psychology, 72, 347-366.

Skitka, L. J., \& Mullen, E. (2002). Understanding judgments of fairness in a real-world political context: A test of the value protection model of justice reasoning. Personality and Social Psychology Bulletin, 28(10), 1419-1429.

Stan Development Team. (2021). RStan: The R interface to Stan. Retrieved September 9, 2021, from http://mc-stan.org/

Steegen, S., Tuerlinckx, F., Gelman, A., \& Vanpaemel, W. (2016). Increasing transparency through a multiverse analysis. Perspectives on Psychological Science, 11(5), 702-712. https://doi.org/10.1177/1745691616658637

Strohminger, N., \& Nichols, S. (2014). The essential moral self. Cognition, 131(1), 159-171.

Tewarson, H., Greene, K., \& Fraser, M. (2021). State strategies for addressing barriers during the early us covid-19 vaccination campaign. American Journal of Public Health, $111(6), 1073-1077$.

Tiu, A., Susswein, Z., Merritt, A., \& Bansal, S. (2021). Characterizing the spatiotemporal heterogeneity of the COVID-19 vaccination landscape. medRxiv. https://doi.org/10.1101/2021.10.04.21263345 
UNICEF. (2021). COVID-19 vaccine market dashboard. Retrieved September 13, 2021, from https://www.unicef.org/supply/covid-19-vaccine-market-dashboard

Vehtari, A., Gelman, A., \& Gabry, J. (2017). Practical Bayesian model evaluation using leave-one-out cross-validation and WAIC. Statistics and Computing, 27(5), 1413-1432. https://doi.org/10.1007/s11222-016-9696-4

Walch, T. (2021). Senior church leaders over 70 vaccinated, urge members to safeguard 'themselves and others through immunization'. Desert News. Retrieved January 19, 2021, from https://www.deseret.com/faith/2021/1/19/22238767/latter-day-saintchurch-leaders-vaccinated-urge-members-to-protect-selves-mormon-lds

Walker, K., \& Herman, M. (2021). tidycensus: Load US census boundary and attribute data as 'tidyverse' and 'sf'-ready data frames. https://CRAN.R-project.org/package=tidycensus

Wang, W., Rothschild, D., Goel, S., \& Gelman, A. (2015). Forecasting elections with non-representative polls. International Journal of Forecasting, 31(3), 980-991. 